\title{
A new module for the tracking of radar-derived precipitation with model-derived winds
}

\author{
T. Winterrath ${ }^{1}$ and W. Rosenow ${ }^{2}$ \\ ${ }^{1}$ German Weather Service, Offenbach a. M., Germany \\ ${ }^{2}$ German Weather Service, Potsdam, Germany \\ Received: 31 July 2006 - Revised: 9 January 2007 - Accepted: 21 February 2007 - Published: 26 April 2007
}

\begin{abstract}
A new approach for the nowcasting of precipitation has been developed at the German Weather Service combining extrapolation techniques and Numerical Weather Prediction (NWP) for a lead time range of several hours. Radarderived precipitation fields serve as input data for a tracking algorithm using model-derived wind data. The composite precipitation field is derived from the precipitation scans which are performed every five minutes at the 16 German radar stations. The data are corrected from clutter and shading effects. The tracking of this radar-derived precipitation field is performed using the temporally and spatially resolved horizontal wind fields at different pressure levels provided by the Local Model Europe (LME). The optimal wind field is derived from minimization of the least-squares difference between a linear combination of model wind data from different pressure levels and the linear displacement vectors calculated via pattern recognition from previous radar measurements. An area-preserving displacement of the precipitation fields is realized by eliminating the wind field divergence and by omitting the dynamical evolution of the precipitation fields. Advection is performed using the fourth-order Bott scheme. Forecasted data comprise precipitation rates for every five minutes lead time as well as hourly sums of precipitation. The verification of a case study's results against radar precipitation measurements lead to a mean Equitable Threat Score (ETS) of $70 \%, 46 \%$, and $38 \%$ for the first, second, and third forecast hour, respectively.
\end{abstract}

Correspondence to: T. Winterrath

(tanja.winterrath@dwd.de)

\section{Introduction}

The accurate prediction of precipitation is still a major task meteorologists are facing today. Forecasting modules range from extrapolation techniques to complex numerical weather prediction (NWP) systems making use of a variety of available observations. Radar nowcasting modules classically base on pattern recognition tools that map similar image structures in successive radar images and thus allow the retrieval of displacement vectors for single precipitation structures. Using the assumption that the movement is static in a Lagrangian sense, the radar image structures' tracks can be extrapolated to the near future. Germann and Zawadzki (2002) and Turner et al. (2004) implement this method using variational echo tracking, a semi-Lagrangian advection scheme, and spatial scale filters to remove structures at lead times larger than their predictability. Li and Lai (2004) apply an object-oriented algorithm to distinguish advection of larger precipitation elements from internal movements of smaller-scale structures. The advantage of radar extrapolation methods is that they start with optimal skill as well as optimal displacement vectors for time $t \rightarrow 0$. However, extracting information on growth and decay of precipitation structures is not straightforward. On the other hand, NWP models start with less skill, mainly determined by data assimilation quality, however, can better capture large-scale movements and the growth and decay of precipitation patterns. Lin et al. (2005) derived a cross-over time between radar extrapolation tools and mesoscale NWP, e.g., the point of time when the scores for NWP get better than the ones for the extrapolation methods, of 6 hours. This cross-over time can be extended by filtering out small-scale structures with shorter lifetimes than the respective lead time. On the other hand, ongoing work on enhancing model resolution along with improving the representation of microphysical processes and data assimilation moves the cross-over time to shorter lead times. Idealistically, the forecasting system combines all available

Published by Copernicus GmbH on behalf of the European Geosciences Union. 
methods and observational data within their most suitable lead time intervals, having in mind the need for fast processing, especially in the nowcasting regime. An approach to combine extrapolation techniques with NWP was presented by Golding (1998). He uses model wind fields in several height levels as well as linear displacement vectors to advect precipitation structures and selects the method that yields the optimal correlation. Radar extrapolation methods and NWP are weighted and merged to produce an optimal forecast.

For the last few years an objective pattern matching forecast procedure using digital satellite and radar data has been operational at the German Weather Service (DWD) (Bartels et al., 2005). This module is part of the "Satellite- and Radar Weather" of DWD. Forecasts are performed for qualitative radar pattern, quantitative rainfall rates from radar reflectivity, clouds and significant weather charts (including lightning). The crucial nowcasting technique is a special pattern recognition program, applied to radar and Meteosat Second Generation satellite data. The results of the forecasts are satisfying up to a lead time of two hours. Nevertheless, there is a strong dependency on the quality of the radar data. In order to extend the forecast to larger lead times a new technique of combining radar-derived displacement vector fields with NWP wind fields has been developed at DWD.

Here, this new approach for the nowcasting of precipitation combining extrapolation techniques and NWP wind fields designed for a lead time range of several hours is presented. The composite precipitation field derived from the precipitation scans which are performed every five minutes at the 16 German radar stations serve as input data. The data are effectively corrected from clutter using a newly-developed filter method. The tracking of this radar-derived precipitation field is performed using the temporally and spatially resolved horizontal wind fields from the Local Model Europe (LME) (Steppeler et al., 2003; Schulz and Schättler, 2005). An areapreserving displacement of the precipitation fields is realized by eliminating the wind field divergence and by omitting the dynamical evolution of the precipitation fields. The best displacement vector field is derived from online comparison of model wind data from different pressure levels with the linear displacement vectors calculated via pattern recognition from previous radar measurements. Here, details of this newly developed method and results of a case study verified against radar measurements are presented. Additionally, the results of the observation-based extrapolation method are shown for comparison.

\section{Data}

\subsection{Radar-derived precipitation fields}

Radar-derived surface precipitation fields, the so-called RZ product of DWD, serve as input data for the tracking algorithm. At each of the 16 German radar stations precipita- tion scans, i.e., radar scans with the lowest possible elevation angle of the radar beam varying between approximately $0.3^{\circ}$ and $2.0^{\circ}$ depending on the surrounding topography, are performed every five minutes. The resulting data are combined to a composite with a pixel side length of $1 \mathrm{~km}$ covering nearly the whole area of Germany. The results of a precipitation scan are regarded as best possible measurement of and therefore representative for surface precipitation. The reflectivity data are corrected, e.g., from shading effects due to obstacles in the line of radar beam propagation and converted to rain rates using an improved Z-R-relationship that takes into account the characteristic of the precipitation event (Bartels et al., 2005).

\subsection{Model-derived wind fields}

The tracking of the radar-derived precipitation field is performed with a displacement vector field based on the forecasted wind fields of the Local Model Europe (LME), i.e., the operational numerical weather prediction (NWP) high-resolution regional model of DWD. The LME is a non-hydrostatic, compressible atmospheric model. It has $665 \times 657$ grid points in the horizontal with a grid length of $0.0625^{\circ}$, i.e., appoximately $7 \mathrm{~km}$, and 40 vertical levels using hybrid coordinates. Wind fields are given on distinct pressure levels. In the framework presented here, wind vectors on the pressure levels $500 \mathrm{hPa}, 600 \mathrm{hPa}, 700 \mathrm{hPa}$, and $850 \mathrm{hPa}$ are used. The different pressure levels are weighted in an Optimal Linear Combination (OLC) described in detail in Sect. 3.2. To use the wind field as input data it has to be interpolated to the radar pixel field. Interpolation is realized by taking the mean of the values at the four nearest model grid points weighted with their inverse distance to the respective radar pixel center. Additionally, a coordinate transformation of the wind vectors is applied.

\subsection{Radar-derived displacement vector field}

Additional information for the advection of the precipitation fields is taken from the observation-based extrapolation module, in the following referred to as OBE, that has been run operationally at DWD for several years (Bartels et al., 2005). Precipitation elements are advected using a displacement vector field that is derived from observed data itself. The crucial nowcasting technique is a special pattern recognition program, applied on clutter-filtered radar data covering Central Europe with a spatial resolution of one to two kilometres and a temporal resolution of five to fifteen minutes making additional use of Meteosat Second Generation satellite data. The focus lies on the meso- $\beta$ scale covering predominantly stratiform precipitation fields, however extension to the meso- $\gamma$ scale is underway to additionally cover convective shorter-lived structures. The displacement vector field is derived from the mapping of recognized precipitation structures in successive picture data. The resulting 
displacement vectors are interpolated and a weighted mean of the actual data and the vector fields of the preceeding two time steps is generated to guarantee a steady displacement over time. Thus, in every timestep a displacement vector field is generated that reflects the real track for times $t<0$ and, thus, is a good approximation for small $t$. This vector field is regarded as the optimal solution at the location of the precipitation at starting time.

\section{Technique}

\subsection{Clutter filter algorithm}

\subsubsection{Motivation}

Radar measurements are subject to multiple sources of error that lead to artificial precipitation pixels in the radar product. These false echoes are largely eliminated in the operational process, however, to prevent removal of any true precipitation structures from the data the applied methods are conservative leaving some clutter pixels in the radar product. Due to the inherent numerical diffusion of Eulerian transport schemes, single high-intensity pixels are smeared out making them less recognizable as clutter pixels in the forecast product. Therefore, an additional filter algorithm has been developed that effectively eliminates all clutter pixels within the composite data.

\subsubsection{Method}

The basic assumption of the presented approach is that clutter pixels appear scattered with varying magnitudes, whereas precipitation pixels are more clustered within a homogeneous precipitation field. Technically, each pixel is scanned individually and marked as clutter pixel, if a certain threshold percentage of pixels within a $31 \times 31$ surrounding square has significantly smaller values than the respective central pixel, i.e., less than $45 \%$ of the central pixel's value. The threshold percentage taken in this studies is $85 \%$. In areas, in which clutter pixels are observed regularly, e.g., close to the radar stations, the value is lowered to $60 \%$ to enhance the filtering effectivity. In a second step, the identified clutter pixels are either removed in non-rainy areas or, when the clutter pixel of high intensity is masked by a precipitation field, set to the mean value of all non-clutter pixels within the surrounding square.

This method shows good results in effectively eliminating clutter pixels with negligible effect on precipitation structures (Bartels et al., 2005).

\subsection{Optimal Linear Combination (OLC)}

Considering the displacement vectors at the location of the precipitation elements the best estimate at start time, the best wind field based on model-derived winds is that with the smallest difference to the observation-based vector field. To generate an optimal displacement vector field based on the model wind data, we use a linear combination of the wind fields in $n$ different pressure levels:

$u_{i j}=\alpha_{p_{1}} u_{i j, p_{1}}+\ldots+\alpha_{p_{n}} u_{i j, p_{n}}$

$v_{i j}=\beta_{p_{1}} v_{i j, p_{1}}+\ldots+\beta_{p_{n}} v_{i j, p_{n}}$

with $n=4$ and $p_{1}=850 \mathrm{hPa}, p_{2}=700 \mathrm{hPa}, p_{3}=600 \mathrm{hPa}$, $p_{4}=500 \mathrm{hPa}$ taken in this paper. The coefficients of the Optimal Linear Combination (OLC) are derived via minimization of the squared difference $S$ between the observation-based vector field $\left[u_{i j}^{\mathrm{obs}}, v_{i j}^{\mathrm{obs}}\right]$ and the linear combination of the model wind fields $\left[u_{i j}, v_{i j}\right]$ at those pixels $[i, j]$ at which the rain rate $R R_{i j}$ is non-zero and, thus, the pattern recognition algorithm provides displacement vectors:

$S=\sum_{\substack{i, j=1 \\ \wedge R R_{i j} \neq 0}}^{N}\left[\left(u_{i j}-u_{i j}^{\mathrm{obs}}\right)^{2}+\left(v_{i j}-v_{i j}^{\mathrm{obs}}\right)^{2}\right] \stackrel{!}{=} \operatorname{Min}$

with $N=900$ being the number of pixels in each horizontal direction of the radar composite data field. By setting the derivatives to zero:

$\frac{\partial S}{\partial \alpha_{p_{1}}}=\frac{\partial S}{\partial \alpha_{p_{2}}}=\ldots=\frac{\partial S}{\partial \beta_{p_{n}}} \stackrel{!}{=} 0$

one gets two linear equation systems each consisting of $n=4$ equations with $n=4$ unknown coefficients $\alpha_{p}$ and $\beta_{p}$, respectively:

$$
\begin{gathered}
\sum_{\substack{i, j=1 \\
\wedge R R_{i j} \neq 0}}^{900}\left[u_{i j, p_{1}} \sum_{k=1}^{4} \alpha_{p_{k}} u_{i j, p_{k}}\right]=\sum_{\substack{i, j=1 \\
\wedge R R_{i j} \neq 0}}^{900} u_{i j}^{\mathrm{obs}} u_{i j, p_{1}} \\
\sum_{\substack{i, j=1 \\
\wedge R R_{i j} \neq 0}}^{900}\left[v_{i j, p_{4}} \sum_{k=1}^{4} \beta_{p_{k}} v_{i j, p_{k}}\right]=\sum_{\substack{i, j=1 \\
\wedge R R_{i j} \neq 0}}^{900} v_{i j}^{\mathrm{obs}} v_{i j, p_{4}}
\end{gathered}
$$

that is solved using a Gauss algorithm. The retrieval of a new displacement vector field is performed once an hour.

\subsection{Elimination of divergence}

In its present form, the module is an exclusive tracking mechanism that neglects the growth or decay of precipitation elements. Thus, it is desirable to perform an area-preserving displacement to inhibit the creation of artificial structures. For this purpose, divergences in the horizontal wind field are eliminated via minimization of the function (Sherman, 1978):

$$
\begin{aligned}
E(u, v, \lambda) & =\int_{A}\left[\alpha^{2}\left(u-u^{0}\right)^{2}+\alpha^{2}\left(v-v^{0}\right)^{2}\right. \\
& \left.+\lambda\left(\frac{\partial u}{\partial x}+\frac{\partial v}{\partial y}\right)\right] d x d y
\end{aligned}
$$


RZ on 29/04/2006, 19:15 UTC

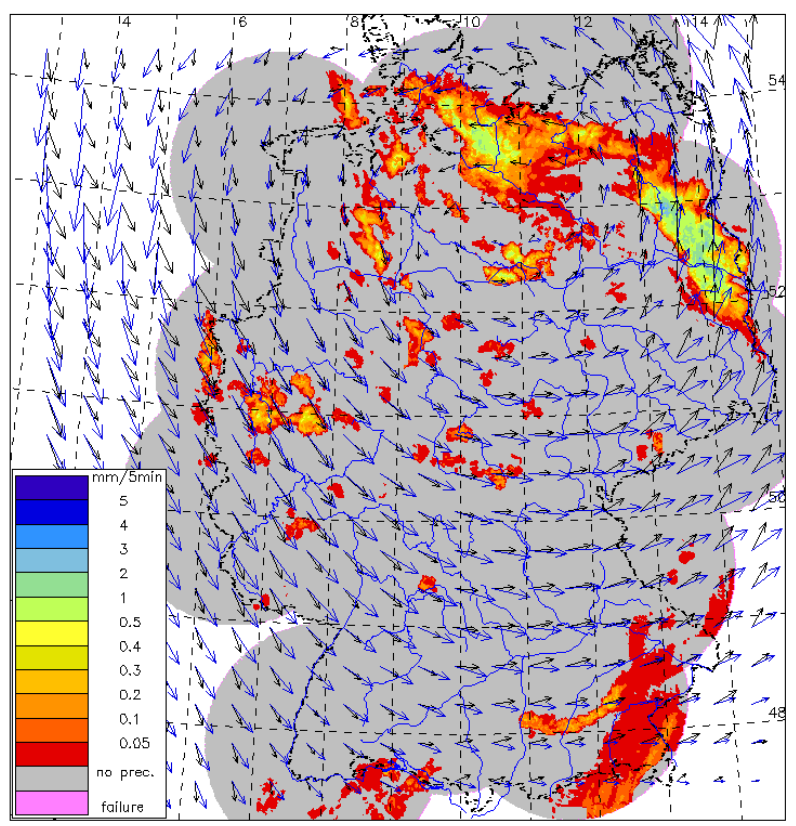

Fig. 1. Rain rate at 19:15 UTC based on the 5-min radar measurement (RZ) with clutter filtering applied; black arrows show the wind field based on the OLC method, blue arrows show the displacement vector field derived from the observations.

where $\alpha$ is the Gauss precision modulus, $\lambda$ is the Langrangian multiplyer, $u^{0}$ and $v^{0}$ are the initial wind components, and $u$ and $v$ are the resulting wind components of the divergencefree wind field. The minimization problem (Eq. 6) is solved iteratively using a Gauss-Seidel algorithm with Successive Over Relaxation and Chebyshev acceleration (Press et al., 1992). In case studies, the elimination of divergence lead to differences in the mean wind speed between the resulting wind fields and the original data of approximately $10 \%$, thus the main advective characteristics of the wind fields are retained.

\subsection{Advection}

The advection of the radar-derived precipitation fields is performed using the fourth-order monotone advection scheme of Bott (1993). The scheme has been proven to produce negligible numerical diffusion, while performing satisfactorily fast. The timestep is determined by the maximum wind speed within the lead time frame and the grid spacing, complying with the Courant-Friedrich-Levy condition. Additionally, the background mask that marks the area in which radar data is available within the $900 \times 900$-pixel area is advected using a fifth-order upwind scheme.

\section{Case Study}

A case study has been performed for 29 April 2006. The weather situation on that day was characterized by an upperlevel cut-off low over the northern part of Germany. At ground level cold marine air was advected from the north. In the area of the upper-level cold air mass convective precipitation associated with rain, partly graupel and snow down to $500 \mathrm{~m}$ was initiated, while in the south-east and north-east stratiform rain dominated triggered by advection of warm air and vorticity.

Figure 1 shows the rain rate at 19:15 based on the 5-min radar measurement, the so-called RZ product, with clutter filtering applied. This data serves as input data for the tracking module. Additionally, the black arrows mark the wind field derived with the OLC method and the blue arrows show the displacement vector field derived from the observations, both valid at 19:15. Although the OLC technique is based on the observation-derived vector field within the precipitation areas both vector fields show significant differences. Large differences can be found outside of the precipitation areas and may become significant for larger lead times.

A stratiform precipitation field with maximum values up to approximately $20 \mathrm{~mm} / \mathrm{h}$ is located in the north-eastern part of Germany moving to the north-west in a counter-clockwise turn. Over the western and mid part of Germany numerous small precipitation areas caused by convective cells are advected to the south-east following the equivalent counterclockwise streamlines opposite of the stratiform elements with respect to the turning center.

Figure 2, left panel, shows the hourly sum of precipitation, the so-called RH-product, between 20:15 and 21:15 UTC based on 5-minute radar measurements with clutter filtering applied. While the stratiform system has moved, some convective elements with their shorter lifetimes have already disappeared. The corresponding results of the tracking algorithms, the so-called RS-products, are shown in the middle (OLC) and right (OBE) panel of Fig. 2. The presented hourly sum valid between 20:15 and 21:15 is based on the 120-min forecast starting at 19:15. The turning movement is very well captured by both algorithms for the stratiform as well as for the convective elements, however, the precipitation caused by the convective cells is overestimated. This is due to the fact that no dissipation can be represented in the actual development stage of the modules. The results of the two modules concerning the direction and velocity of the precipitation fields' movement is similar. The result of the OLC method (Fig. 2, middle panel) shows smaller areas of precipitation, especially valid for those with low intensity. This is due to, first, the more intense clutter filter and, second, the smaller time step used for the advection leading to larger smoothing along the forecasted tracks. This effect can also be seen in the categorical scores. 



Fig. 2. Left: Hourly sum of precipitation (RH) between 20:15 and 21:15 UTC based on 5-min radar measurements with clutter filtering applied; Middle: Hourly forecasted sum of precipitation (RS) between 20:15 and 21:15 UTC based on the tracking of RZ measured at 19:15 (Fig. 1) performed with the Optimal Linear Combination (OLC) technique; Right: Same as middle panel but performed with observationbased extrapolation (OBE)

\subsection{Scores}

Verification of the forecast is performed using categorical scores based on a pixelwise yes-no comparison between the forecasted and the radar-derived hourly sums RS and RH, respectively. The definition of $a$ to $h$ and $N$ are given in Table 1 . The scores used in the following are the Probability of Detection:

$\mathrm{POD}=\frac{d}{h}$,

i.e., the fraction of correctly forecasted rain pixels to all rain pixels with values between 0 (worst) and 100\% (best), the False Alarm Ratio:

$\mathrm{FAR}=\frac{c}{f}$,

i.e., the fraction of falsely forecasted rain pixels to all forecasted rain pixels with values between 0 (best) and $100 \%$ (worst), and the Equitable Threat Score:

ETS $=\frac{d-D_{r}}{c+h-D_{r}} \quad$ with $\quad D_{r}=\frac{f h}{N}$

with $D_{r}$ being the randomly correct forecasted wet points. Values of the ETS range between $-1 / 3$ (worst) and $100 \%$ (best) with 0 being the value of the random forecast.

\subsection{Results}

Forecasts have been performed starting every $30 \mathrm{~min}$ between 18:15 and 19:15 UTC and have generated three hourly sums of precipitation each, one valid for a lead time of 0 to $60 \mathrm{~min}(\mathrm{vv}=60 \mathrm{~min})$, one between 60 and
Table 1. Contingency table for the categorical scores ("Yes": $R R_{i j} \geq 0.01 \mathrm{~mm} / \mathrm{h}$; "No": $R R_{i j}<0.01 \mathrm{~mm} / \mathrm{h}$ ).

\begin{tabular}{ccccc}
\hline & & & Measured & \\
& & No & Yes & Sum \\
\hline \multirow{3}{*}{ Forecasted } & No & $a$ & $b$ & $e$ \\
& Yes & $c$ & $d$ & $f$ \\
& Sum & $g$ & $h$ & $N$ \\
\hline
\end{tabular}

$120 \mathrm{~min}(\mathrm{vv}=120 \mathrm{~min})$, and one between 120 and $180 \mathrm{~min}$ ( $\mathrm{vv}=180 \mathrm{~min})$. The OBE method is limited to a two-hours forecast.

A qualitative assessment of the forecast has already been given in the introduction to this chapter. A more quantitative evaluation of the forecast is expressed by the categorical scores introduced in Sect. 4.1. Table 2 gives the POD, FAR, and ETS for the three forecast time ranges for 3 model simulations performed with the OLC technique presented in this paper and, for comparison, the results of the OBE method. The model results corresponding to Fig. 2 are highlighted. The absolut values of the scores do not show significant variations between the different model start times. While the POD shows high values for all forecast hours, the FAR increases significantly between the first and the second forecast hour dominating the decrease of the ETS. The advection of the stratiform elements is well captured by the algorithms expressed by the high POD values, even for the 180min forecast. On the other hand, the location error in the forecast of convective elements as well as the lack of dynam- 
Table 2. Categorical scores for different start and lead times for the two nowcasting techniques (OLC $=$ Optimal Linear Combination, $\mathrm{OBE}=$ observation-based extrapolation), the 180-min forecast is only available for OLC, the bold numbers correspond to the presented figure.

\begin{tabular}{cccccc}
\hline Module & $\begin{array}{c}\text { Start time } \\
\text { UTC }\end{array}$ & $\begin{array}{c}\text { Lead time } \\
{[\mathrm{min}]}\end{array}$ & $\begin{array}{c}\text { POD } \\
{[\%]}\end{array}$ & $\begin{array}{c}\text { FAR } \\
{[\%]}\end{array}$ & $\begin{array}{c}\text { ETS } \\
{[\%]}\end{array}$ \\
\hline OLC & $18: 15$ & 60 & 83.3 & 16.4 & 71.3 \\
OBE & $18: 15$ & 60 & 87.5 & 21.4 & 70.3 \\
OLC & $18: 15$ & 120 & 72.4 & 45.3 & 45.2 \\
OBE & $18: 15$ & 120 & 79.6 & 46.5 & 46.4 \\
OLC & $18: 15$ & 180 & 72.0 & 54.8 & 36.9 \\
OLC & $18: 45$ & 60 & 82.9 & 20.1 & 68.1 \\
OBE & $18: 45$ & 60 & 88.2 & 24.3 & 68.9 \\
OLC & $18: 45$ & 120 & 72.6 & 44.9 & 44.1 \\
OBE & $18: 45$ & 120 & 80.4 & 48.3 & 45.4 \\
OLC & $18: 45$ & 180 & 69.0 & 55.7 & 36.9 \\
OLC & $19: 15$ & 60 & 83.8 & 18.6 & 69.6 \\
OBE & $19: 15$ & 60 & 89.7 & 25.8 & 67.8 \\
OLC & $\mathbf{1 9 : 1 5}$ & $\mathbf{1 2 0}$ & $\mathbf{7 3 . 7}$ & $\mathbf{4 0 . 6}$ & $\mathbf{4 9 . 3}$ \\
OBE & $\mathbf{1 9 : 1 5}$ & $\mathbf{1 2 0}$ & $\mathbf{8 1 . 0}$ & $\mathbf{4 7 . 0}$ & $\mathbf{4 6 . 1}$ \\
OLC & $19: 15$ & 180 & 65.9 & 52.2 & 39.8 \\
OLC & Mean & 60 & 83.3 & 18.4 & 69.7 \\
OBE & Mean & 60 & 88.5 & 23.8 & 69.0 \\
OLC & Mean & 120 & 72.9 & 43.6 & 46.2 \\
OBE & Mean & 120 & 80.3 & 47.3 & 46.0 \\
OLC & Mean & 180 & 69.0 & 54.2 & 37.9 \\
& & & & &
\end{tabular}

ical evolution like, in this case, the dissipation of convective elements are associated with high FAR values in this pixelwise approach, especially pronounced in the two- and threehour forecast. The values for the OBE technique show higher values for both the POD as well as the FAR resulting in an ETS with negligible differences to the OLC method complying with the differences in precipitation areas discussed in the introduction to Sect. 4.

\section{Conclusions}

A new module for the tracking of radar-derived precipitation fields has been developed and experimentally applied at DWD. The radar data used as input data are based on the Germany composite consisting of the precipitation scans of the 16 German radar stations. The data are converted to rain rates using an improved Z-R relationship. Clutter pixels are effectively removed using a new filter, while precipitation structures remain largely unchanged. The displacement vector field used for the advection is based on a linear combination of the horizontal wind fields at different pressure levels of the Local Model Europe, i.e., the operational NWP model of DWD. The coefficient for the optimal linear combination (OLC) are derived via the minimization of the squared differences to the displacement vector field of the observation-based extrapolation module (OBE) that has been operational at DWD for several years. The latter field is extracted from successive radar images via a pattern recognition algorithm. Advection is performed using a fifth-order upwind scheme for the background and the fourthorder monotone Bott scheme for the precipitation fields.

The presented case study reveals the potential of the module to realistically track stratiform and convective precipitation fields. The dynamical evolution of precipitation, however, cannot be reproduced by the module in its present stage of development. Standard categorical scores are calculated to quantify the predictive quality of the module and to allow an online comparison to other nowcasting systems like the observation-based displacement module that is currently operationally in use at DWD. No significant differences between the two forecast methods can be found for the presented case study reflected in similar ETS values. Due to different time steps and filter algorithms the OBE technique tends to forecast larger precipitation areas than the OLC method resulting in higher POD as well as FAR.

A long-term comparison study between the two methods has been initiated and will be continued operationally at DWD. Besides average forecast quality, performance and reliability are important criteria for the evaluation of the nowcasting module. Using radar data for the extraction of displacement vectors, the forecast method depends on the quality of the radar data. On the other hand, the OBE technique performs much faster and is well suited for operational nowcasting. Therefore, the overall aim will be to draw the benefits from both methods to improve the quality and reliability of precipitation nowcasting at DWD.

Acknowledgements. T. Winterrath thanks J. Förstner for providing the upwind scheme and for helpful discussions on the numerics, A. Bott for making the advection algorithm available, D. Majewski for initiating the OLC method, and T. Reich for providing the plotting routine. This work was funded by the Aktionsprogramm 2003 of DWD.

Edited by: S. C. Michaelides and E. Amitai

Reviewed by: anonymous referees

\section{References}

Bartels, H., Weigl, E., Klink, S., Kohler, O., Reich, T., Rosenow, W., Lang, P., Podlasly, C., Winterrath, T., Adrian, G., Majewski, D., and Lang, J.: Projekt RADVOR-OP: Radargestützte, zeitnahe Niederschlagsvorhersage für den operationellen Einsatz (Niederschlag-Nowcasting-System), Final Report, Deutscher Wetterdienst, available at: http: //www.dwd.de/de/wir/Geschaeftsfelder/Hydrometeorologie/ a_href_pages/RADVOR-OP/Abschlussbericht.pdf, 2005.

Bott, A.: The monotone area-preserving flux-form advection algorithm: Reducing the time-splitting error in two-dimensional flow fields, Mon. Wea. Rev., 121, 2637-2641, 1993. 
Germann, U. and Zawadzki, I.: Scale dependence of the predictability of precipitation from continental radar images. Part I: Description of the methodology, Mon. Wea. Rev., 130, 2859-2873, 2002.

Golding, B. W.: Nimrod: A System for Generating Automated Very Short Range Forecasts, Meteorol. Appl., 5, 1-16, 1998.

Li, P. W. and Lai, E. S. T.: Short-Range Quantitative Precipitation Forecast in Hong Kong, J. Hydrol., 288, 189-209, 2004.

Lin, C., Vasic, S., Kilambi, A., Turner, B., and Zawadzki, I.: Precipitation forecast skill of numerical weather prediction models and radar nowcasts, Geophys. Res. Lett., 32, L14 801, doi:10.1029/2005GL023 451, 2005.

Press, W. H., Teukolsky, S. A., Vetterling, W. T., and Flannery, B. P.: Numerical Recipes in Fortran: The Art of Scientific Computing, Cambridge University Press, 2 edn., 1992.
Schulz, J.-P. and Schättler, U.: Kurze Beschreibung des LokalModells LME und seiner Datenbanken auf dem Datenserver des DWD, Tech. rep., Deutscher Wetterdienst, 2005.

Sherman, C. A.: A mass-consistent model for wind fields over complex terrain, J. Appl. Meteor., 17, 312-319, 1978.

Steppeler, J., Doms, G., Schättler, U., Bitzer, H. W., Gassmann, A., Damrath, U., and Gregoric, G.: Meso-gamma scale forecasts using the nonhydrostatic model LM, Meteorol. Atmos. Phys., 82, 75-96, 2003.

Turner, B. J., Zawadzki, I., and Germann, U.: Predictability of Precipitation from Continental Radar Images. Part III: Operational Nowcasting Implementation (MAPLE), J. Appl. Meteor., 43, 231-248, 2004. 